\title{
Assessment of Procedure Related Anxiety and Depression in Oncologic Patients before F-18 FDG PET-CT Imaging
}

Umut Elboga ${ }^{1^{*}}$, Gulcin Elboga ${ }^{2}$, Ceyhun Can ${ }^{3}$, Ertan Sahin ${ }^{4}$, Huseyin Karaoglan ${ }^{1}$, Ebuzer Kalender ${ }^{1}$, Hasan Deniz Demir ${ }^{1}$, Mustafa Basibuyuk ${ }^{1}$ and Y Zeki Celen $^{1}$

${ }^{1}$ Department of Nuclear Medicine, Gaziantep University, Gaziantep, Turkey

${ }^{2}$ Department of Psychiatry, Gaziantep University, Gaziantep, Turkey

${ }^{3}$ Department of Psychiatry, Duzıcı State Hospital, Osmaniye, Turkey

${ }^{4}$ Department of Nuclear Medicine, Namik Kemal University, Tekirdag, Turkey

*Corresponding author: Umut Elboga, Gaziantep University, School of Medicine, Department of Nuclear Medicine, Gaziantep, Turkey, Tel: +90-342-360 60 60; Fax +90-342-360 39 28; E-mail: umutelboga@hotmail.com

Received Date: October 14, 2014, Accepted Date: November 25, 2014, Published Date: December 03, 2014

Copyright: @ 2015 , Umut Elboga, et al., This is an open-access article distributed under the terms of the Creative Commons Attribution License, which permits unrestricted use, distribution, and reproduction in any medium, provided the original author and source are credited.

\begin{abstract}
Background: The aim of this study was to study objectively the level of anxiety and depression in patients undergoing positron emission tomography-computed tomography (PET-CT). One hundred and forty four oncologic out-patients (76 male, 68 female) were included in this study.

Methods: All patients were referred to Nuclear Medicine Department for Fluorine-18 fluorodeoxyglucose (F-18 FDG) PET-CT imaging for the assessment of their malignant or possibly malignant diseases. The Hospital Anxiety Depression Scale and the State and Trait Anxiety Inventory I and II were used to evaluate the anxiety and depression levels in these patients.

Results: The mean anxiety and depression scores of The Hospital Anxiety Depression Scale prior to F-18 FDG PET-CT were $9.2( \pm 3.8)$ and $6.6( \pm 3.4)$, respectively. The mean state and trait anxiety scores of the State and Trait Anxiety Inventory I and II prior to F-18 FDG PET-CT were 40.4 ( \pm 8.5$)$ and $46.62 \pm 7.8$, respectively. The Hospital Anxiety Depression Scale and the State and Trait Anxiety Inventory I and II anxiety scores were found to be significantly higher in female patients, smokers and in patients with higher stage disease.
\end{abstract}

Conclusion: Our results suggest that F-18 FDG PET-CT imaging may at least contribute to patient's baseline anxiety which is already generated by being an oncology patient, and thus nuclear medicine physicians should handle the patients with extra care to minimize this affect.

Keywords: Anxiety; Depression; Oncologic outpatients; PET-CT

\section{Introduction}

Fluorine-18 fluorodeoxyglucose positron emission tomographycomputed tomography (F-18 FDG PET-CT) is an efficient and clinically useful tool for the diagnosis, staging and restaging as well as the assessment of response to anti-neoplastic treatment in many malignant diseases. Patients with known malignant diseases often suffer from psychiatric comorbidities, thereby significantly decreasing their quality of life. Mitchell et al reported that $47 \%$ of patients with malignant diseases have psychiatric disorders [1]. However, only $0.5-3 \%$ of these patients are referred to psychiatrists and many patients with anxiety symptoms or depression are presumably overlooked. The prediction, early detection and intervention of depressive and anxiety symptoms would therefore improve the life quality in patients with malignant diseases [2].

Anxiety and depression caused by invasive and non-invasive diagnostic procedures including magnetic resonance imaging was previously reported, but, to the best of authors' knowledge, there is no study published yet describing anxiety and depression possibly caused by PET/CT procedure [3-5].
We hypothesized that patients undergoing F-18 FDG PET-CT scan may experience additional anxiety and depression due to the anticipation of findings and concern about the PET/CT procedure. The aims of this prospective study were therefore to evaluate objectively procedure related anxiety and depression before PET/CT imaging in order to determine whether FDG PET-CT procedure causes anxiety and depression in patients with already diagnosed malignant or possibly malignant disease before F-18 FDG PET-CT procedure, and to correlate the results with other risk factors including previous oncologic surgery, radiotherapy and/or chemotherapy, stage of the malignant disease, smoking and perceived body image.

\section{Material and Methods}

The current study was conducted at Gaziantep University Hospital, Nuclear Medicine Department, Turkey, where patients are referred from oncology departments for assessment of their malignant diseases. Gaziantep University Hospital is a governmental and referral hospital. In this study, one hundred and forty-four oncologic outpatients suffering from cancer during April to May 2013. Hospital Anxiety Depression Scale and the State and Trait Anxiety Inventory I and II were used to evaluate the anxiety levels in these eligible patients. Finally, all patients were informed of the nature of the study and were 
asked to provide informed consent. Patients were judged eligible to enter the study if they fulfilled the following inclusion criteria: histologically confirmed malignancy, over 18 years old, ability to communicate effectively with the study personnel, and knowledge of the disease diagnosis. Exclusion criteria were the following: a diagnosis of psychotic illness and significant cognitive impairment.

\section{Anxiety assessment}

The Hospital Anxiety Depression Scale and the State and Trait Anxiety Inventory I and II were used to evaluate the anxiety levels in these patients. The patients were interviewed and the psychological tests (the Hospital Anxiety Depression Scale and the State and Trait Inventory I and II) were applied in one session by a psychiatrist before the injection of F-18 FDG at the Nuclear Medicine Department [6,7]. The Hospital Anxiety Depression Scale scores were evaluated between 0 and 21 points; patients with $8-10$ points were classified as having 'tendency to anxiety' and 10 or higher points as 'anxiety' [7].

\section{PET-CT imaging protocol}

All patients were imaged with the same scanner PET-CT (Biograph Duo, Siemens). After a 6-hour fasting period, patients were injected intravenously with $296-370 \mathrm{MBq}(8-10 \mathrm{mCi})$ of F18-FDG. Imaging was initiated after an uptake period, during which patients were encouraged to rest in a long armchair. The total waiting time varied between 55 and 95 minutes, acquisition time between 25 and 35 minutes per patient.

\section{Statistical analysis}

The prevalence of anxiety was described by point estimation and the 95\% confidence interval (CI 95\%). Associations between anxiety and demographical, clinical, and PET-CT variables were calculated by the chi-square association test. For all tests, an error $\alpha=5 \%$ was established. The Institution Ethics Committee approved the study protocol (date: 01/08/2013 and number: 21).

\section{Results}

The mean age was 62.6 years for the whole cohort of patients, 63.8 for the female patients, 61.2 for the male patients (Table 1). Of the 144 participants, $77(53.4 \%)$ were 55 years or older. The diagnosis of malignancy had been made more than one year earlier in 75 patients (52\%). The most common malignancies were lung carcinoma (43\%), lymphoma $(28.4 \%)$, breast carcinoma (13.8\%), thyroid carcinoma (\%11.1) and colon carcinoma (3.7\%). Seventy seven patients $(53.4 \%)$ were aware of their disease, and $31.9 \%$ had an additional chronic disease (such as hypertension, respiratory disorders, diabetes or arthritis).

Depression and anxiety scores and state-trait anxiety scores are listed in Tables 2-4. The mean anxiety and depression scores of The Hospital Anxiety Depression Scale prior to PET-CT were 9.2 ( \pm 3.8 ) and $6.6( \pm 3.4)$, respectively. The mean state and trait anxiety scores of the State and Trait Anxiety Inventory I and II prior to PET-CT were $40.4( \pm 8.5)$ and $46.62 \pm 7.8$, respectively $(\mathrm{p}=0.007$ and $\mathrm{p}=0.011)$. The Hospital Anxiety Depression Scale and the State and Trait Anxiety Inventory I and II anxiety scores were found to be significantly high in female patients $(\mathrm{p}=0.000)$, smokers $(\mathrm{p}=0.031)$ and patients with higher stage disease $(\mathrm{p}=0.016)$. No significant difference was found between the The Hospital Anxiety Depression Scale and the State and Trait
Anxiety Inventory I and II scores. Previous surgery, chemotherapy and/or radiotherapy and perceived body image were not related to depression and anxiety scores $(\mathrm{p} \geq 0.05)$.

\begin{tabular}{|c|c|c|}
\hline Independent variable & Characteristic & $\begin{array}{l}\% \\
\text { participants }\end{array}$ \\
\hline Average age & 62.6 years & $\mathrm{NAa}$ \\
\hline \multirow[t]{2}{*}{ Gender } & Female & 45.9 \\
\hline & Male & 54.1 \\
\hline \multirow[t]{2}{*}{ Marital status } & Single & 16.7 \\
\hline & Married & 83.3 \\
\hline \multirow[t]{3}{*}{ Income } & High & 5.5 \\
\hline & Average & 56.2 \\
\hline & Low & 38.3 \\
\hline \multirow[t]{2}{*}{ Education level } & Primary school & 47.9 \\
\hline & >Primary school & 52.3 \\
\hline \multirow[t]{2}{*}{ Have children } & Yes & 35.4 \\
\hline & No & 64.6 \\
\hline \multirow[t]{5}{*}{ Cancer localization } & Lung Carcinoma & 43 \\
\hline & Lymphoma & 28.4 \\
\hline & Breast Carcinoma & 13.8 \\
\hline & Thyroid Carcinoma & 11.1 \\
\hline & Colon Carcinoma & 3.7 \\
\hline \multirow[t]{2}{*}{ Confirmed cancer } & Yes & 53.4 \\
\hline & No & 46.6 \\
\hline \multirow[t]{2}{*}{ Additional chronic disease } & Yes & 31.9 \\
\hline & No & 68.1 \\
\hline
\end{tabular}

Table 1: Patients' Characteristics

\section{Discussion}

A medical procedure including a diagnostic imaging test is perceived by patients differently than medical professionals, particularly when a patient undergoes a procedure for the first time. Being a patient alone generates anxiety, and PET-CT procedure or being scheduled for it would contribute above the baseline anxiety state in these patients. Psychological reactions, ranging from slight apprehension to severe anxiety were previously reported during and before invasive and non-invasive medical procedures [8-11]. The anxiety associated with medical procedures originates from i) fear of the unknown, ii) anticipation of discomfort, iii) concerns about diagnosis and prognosis, iv) lack of control during the procedure [12].

\begin{tabular}{|l|l|l|l|}
\hline & Female/Male & Mean \pm Sd & $\mathbf{p}$ \\
\hline HAD anxiety & $68 / 76$ & $9.1 \pm 3.6 / 5.96 \pm 3.9$ & $0.000^{*}$ \\
\hline HAD depression & $68 / 76$ & $6.90 \pm 3.7 / 10.67 \pm 3.8$ & $0.011^{* *}$ \\
\hline STAI state & $68 / 76$ & $45.40 \pm 9.8 / 38.34 \pm 10.06$ & $0.000^{*}$ \\
\hline STAI trait & $68 / 76$ & $47.42 \pm 7.04 / 42.91 \pm 8.96$ & $0.000^{*}$ \\
\hline
\end{tabular}

Table 2: Hospital Depression and Anxiety scores and State-Trait Anxiety scores, ${ }^{* *} \mathrm{p}<0.005,{ }^{*} \mathrm{p}<0.001$ 


\begin{tabular}{|l|l|l|l|}
\hline & Smoker/Non-smoker & Mean \pm Sd & $\mathbf{p}$ \\
\hline HAD anxiety & $77 / 67$ & $7.76 \pm 3.3 / 8.4 \pm 2.9$ & $0.000^{*}$ \\
\hline $\begin{array}{l}\text { HAD } \\
\text { depression }\end{array}$ & $77 / 67$ & $4.7 \pm 2.4 / 7.3 \pm 3.6$ & $0.009^{\star \star}$ \\
\hline STAl state & $77 / 67$ & $\begin{array}{l}38.12 \pm 9.6 / 41.4 \pm \\
10.2\end{array}$ & $0.000^{\star}$ \\
\hline STAI trait & $77 / 67$ & $42.4 \pm 7.3 / 46.9 \pm 8.8$ & $0.000^{*}$ \\
\hline
\end{tabular}

Table 3: Hospital Depression and Anxiety scores and State-Trait Anxiety scores in smokers and non-smokers, ${ }^{* *} \mathrm{p}<0.005,{ }^{*} \mathrm{p}<0.001$

\begin{tabular}{|l|l|l|l|}
\hline & $\begin{array}{l}\text { Higher/Earlier Stage } \\
\text { Patients }\end{array}$ & Mean \pm Sd & $\mathbf{p}$ \\
\hline HAD anxiety & $46 / 98$ & $5.96 \pm 3.8 / 8.8 \pm 3.3$ & $0.000^{*}$ \\
\hline $\begin{array}{l}\text { HAD } \\
\text { depression }\end{array}$ & $46 / 98$ & $7.90 \pm 3.5 / 10.4 \pm 3.5$ & $0.008^{* *}$ \\
\hline STAl state & $46 / 98$ & $45.2 \pm 9.8 / 42.6 \pm 10.6$ & $0.000^{*}$ \\
\hline STAl trait & $46 / 98$ & $\begin{array}{l}43.5 \pm 5.04 / 47.91 \pm \\
9.6\end{array}$ & $0.000^{*}$ \\
\hline
\end{tabular}

Table 4: Hospital depression and anxiety scores and state-trait anxiety scores in higher stage and lower stage patients. ${ }^{\star *} \mathrm{p}<0.005,{ }^{\star} \mathrm{p}<0.001$

Personality, age, psychiatric history, previous therapeutic and diagnostic experience including surgery, chemotherapy, radiotherapy and perceived body image may however also have additional role on the composition of patient's feelings. It is important for Nuclear Medicine professionals to identify the anxiety and depression possibly caused by PET-CT maging in order to better manage them during imaging procedure.

Our results showed that the anxiety scores of both The Hospital Anxiety Depression Scale and the State and Trait Inventory I and II are moderately high before PET-CT imaging. This level of anxiety is comparable to those that were reported for magnetic resonance imaging [13]. However, 30 patients (21\%) had higher state anxiety scores ( 25 and above). In this subgroup of patients, the mean age was 64.1 years old, $17(56.1 \%)$ were female, $20(66.6 \%)$ were smokers, 2 (6.6\%) had stage I, 3 (9.9\%) stage II, 7 (23.1\%) stage III and 18 (59.4\%) stage IV disease; malignancies included lung carcinoma $(n=13)$, lymphoma $(n=9)$, breast carcinoma $(n=5)$ and colon carcinoma $(n=3)$.

All patients in this study were found to have high trait anxiety levels, which may be partly due to disease-specific conditions rather than PET-CT procedure itself although the diagnosis of malignacy had been made more than one year before PET-CT procedure in 75 patients (52\%). Narita et al. found that there are spesific interactions between high trait anxiety and abnormalities of sympathetic activity [14].

Our results indicate that female patients had higher Hospital Anxiety Depression Scale and the State and Trait Inventory I and II scores suggesting that they are more strongly concerned about PETCT procedure. Previous studies also demonstrated that women have higher anxiety levels during medical procedures as well as in their daily life [15-17].
Contrary to our expectation, no relation was found between the previous therapeutic procedures (i.e. surgery, radiotherapy, chemotherapy) and anxiety and depression scores. Also, no relation was noted between anxiety and depression scores and the patients' perceived body image. Previous studies however, demonstrated that there was significant relation between these factors and anxiety and depression [18-20]. Another unexpected finding is that annxiety and depression scores were significantly higher in smoking patients.

There were significantly higher scores in our patients with higher stage disease, and this was consistent with findings of Vodermaier et al who reported that the stage of malignant disease was directly associated with emotional distress [21].

Although illness provokes anxiety and depression in oncology patients, the increment in anxiety detected in this study may be due to F-18 FDG PET-CT procedure, which, at least, further intensifies the basal state of anxiety in oncologic patients. The Nuclear Medicine physicians in charge of the PET-CT procedure should be aware of the patients' state of mind and prepared to deal with this anxiety in order to give better care to these patients. Some measures including prior interview and reassurance before F-18 FDG injection may prevent worsening of the state of anxiety. Also, psyciatric support in selected cases, especially in female, smoking and high stage patients would be helpful in avoiding high level of anxiety and depression. Other interventions may be suitable such as psychosocial and educational interventions, courses in communication skills for health care professionals, brief psychotherapy, pharmachological therapy [22]. If a brief counselling (or other intervention) before PET-CT may reduce patients anxiety.

The limitation of this study is the lack of a control group. We could have a control group either from patients undergoing non-oncologic PET-CT or oncologic patients who did not undergo PET-CT at all. Our cohort of patients was composed of oncology patients only because cardiac PET, neuro-PET and other clinical applications of PET-CT imaging procedures are not common in our Department, and thus it would not be possible for us to recruit statistically reliable number of patients for a control group. Also, our study design did not allow us to have a control group from patients who did not undergo PET-CT imaging after the initial histological diagnosis of malignancy before being referred to PET-CT because PET-CT imaging was routinely performed in most of the patients in clinical oncology practice during the diagnostic work-up before the histologic diagnosis is made and explained to the patients. Oncologic patients referred to Nuclear Medicine units for PET-CT scan procedure, particularly female patients, smokers and patients with high stage disease, who already have basal anxiety, may have procedure-related anxiety and depression.

\section{References}

1. Mitchell AJ, Ferguson DW, Gill J, Paul J, Symonds P (2013) Depression and anxiety in long-term cancer survivors compared with spouses and healthy controls: a systematic review and meta-analysis. Lancet Oncol 14: 721-732. 
Citation: Elboga U, Elboga G, Can C, Sahin E, Karaoglan H, et al. (2015) Assessment of Procedure Related Anxiety and Depression in Oncologic Patients before F-18 FDG PET-CT Imaging. J Psychiatry 18: 215. doi:10.4172/2378-5756.1000215

Page 4 of 4

2. Uchitomi Y, Fukase M, Sugihara J (1993) Aliason program influences psychiatric consultation rates in cancer patients at a Japanese general hospital. Seventh Annual Meeting of the European Society of Psychosocial Oncology. Abstract p. 99.

3. Higashi TS, Takizawa K, Hasebe S, Takagi T, Yamada M, et al (1991) Prospective evaluation of psychologic effect on incidence of delayed symptoms after intravenous injection of low-osmolar contrast media. Radiology 181:105.

4. Hopper KD, Houts PS, TenHave TR, Matthews YL, Colon E, et al. (1994) The effect of informed consent on the level of anxiety in patients given i.v. contrast material. AJR Am J Roentgenol 162: 531-535.

5. Spring DB, Winfield AC, Friedland GW, Shuman WP, Preger L (1988) Written informed consent for i.v. contrast-enhanced radiography: patients' attitudes and common limitations. AJR Am J Roentgenol 151: 1243-1245.

6. Aydemir O (1997) Validity and Reliability of Turkish Version of Hospital Anxiety and Depression Scale. Turk PsikiyatriDerg. 8: 280-287.

7. Zigmond AS, Snaith RP (1983) The hospital anxiety and depression scale.ActaPsychiatrScand 67: 361-370.

8. McIsaac HK, Thordarson DS, Shafran R, Rachman S, Poole G (1998) Claustrophobia and the magnetic resonance imaging procedure. J Behav Med 21: 255-268.

9. Frenzer A, Binek J, Hammer B (1996) [Physician-patient relations and stress caused by coloscopy in patients with chronic inflammatory bowel disease].Schweiz Med WochenschrSuppl 79: 94S-96S.

10. Kutlutürkan S, Görgülü U, Fesci H, Karavelioglu A (2010) The effects of providing pre-gastrointestinal endoscopy written educational material on patients' anxiety: a randomised controlled trial.Int J Nurs Stud 47: 1066-1073.

11. Carneiro AF, Mathias LA, RassiJúnior A, Morais NS, Gozzani JL, et al. (2009) [Evaluation of preoperative anxiety and depression in patients undergoing invasive cardiac procedures]. Rev Bras Anestesiol 59: 431-438.
12. Horne DJ, Vatmanidis P, CareriA(1994) Preparing patients for invasive medical and surgical procedures. 1: Adding behavioral and cognitive interventions. Behav Med.20:5-13.

13. Grey SJ, Price G, Mathews A (2000) Reduction of anxiety during MR imaging: a controlled trial.MagnReson Imaging 18: 351-355.

14. Narita K, Murata T, Hamada T, Takahashi T, Omori M, et al. (2007) Interactions among higher trait anxiety, sympathetic activity, and endothelial function in the elderly. J Psychiatr Res 41: 418-427.

15. Dantendorfer K, Amering M, Bankier A, Helbich T, Prayer D, et al. (1997) A study of the effects of patient anxiety, perceptions and equipment on motion artifacts in magnetic resonance imaging.MagnReson Imaging 15: 301-306.

16. Luck A, Pearson S, Maddern G, Hewett P (1999) Effects of video information on precolonoscopy anxiety and knowledge: a randomised trial. Lancet 354: 2032-2035.

17. Zender R, Olshansky E (2009) Women's mental health: depression and anxiety.NursClin North Am 44: 355-364.

18. Ozalp E, Cankurtaran ES, Soygür H, Geyik PO, Jacobsen PB (2007) Screening for psychological distress in Turkish cancer patients.Psychooncology 16: 304-311.

19. Bektas AH, Akdemir $\mathrm{N}(2006)$ The assessment of functional status in individual's who have cancer. Turk J Med Sci. 26: 488-499.

20. Mehnert A, Veers S, Howaldt D, Braumann KM, Koch U, et al(2011) Effects of a physical exercise rehabilitation group program on anxiety, depression, body image, and health-related quality of life among breast cancer patients. Onkologie 34: 248-253.

21. Vodermaier A, Linden W, MacKenzie R, Greig D, Marshall C (2011) Disease stage predicts post-diagnosis anxiety and depression only in some types of cancer. Br J Cancer 105: 1814-1817.

22. Galway K, Black A, Cantwell M, Cardwell CR, Mills M, et al. (2012) Psychosocial interventions to improve quality of life and emotional wellbeing for recently diagnosed cancer patients. Cochrane Database Syst Rev 11: CD007064. 